\title{
JUSTIFICATION OF PARAMETERS AND STRUCTURAL SCHEME OF CONCENTRATED FEED DISPENSER
}

\author{
Vasyl Achkevych, Vitaliy Radchuk, Svitlana Potapova, Oleg Zabolotko
}

National University of Life and Environmental Sciences of Ukraine, Ukraine

achkevychv@gmail.com,vitalr@online.ua,svitlapotapova@gmail.com, zaboleg07@ukr.net

\begin{abstract}
The article is devoted to increasing the efficiency of individually dosed distribution feed for cattle in the stall. The article also substantiates the corresponding structural and functional diagram of the dispenser for individual supply concentrate to cows in accordance with zootechnical requirements. The expediency of dosing compound feed using a volumetric drum-type batcher with a vertical axis of rotation has been analytically substantiated. Theoretical studies of the efficiency of the main parameters of the drum batcher on the indicators of the efficiency of the feed distribution process have been performed. Mathematical models of the accuracy of the drum batcher and the norms of individual feed supply have been obtained. Test studies of the vulnerability of dispensing and unevenness of the main parameters of the dispenser (number of pins, feed rate $V_{k}$, discharge opening angle $\beta$ ) have been carried out. For experimental studies, a laboratory dosing device for individual dosing of feed has been installed. As a result of the experimental studies, a regression equation for the weight of feed given to one cow and deviation from a given norm was obtained. The analysis of regression ratios showed that with a decrease in the feed rate, the difference between the feed in one cell and the rate decreases. This leads to an increase in the output error. When the feed rate increases, the feed from the cells does not have time to fall when passing over the discharge opening. The rational parameters of the dispenser have been determined: the value of the speed will be $V=0.2 \mathrm{~m} \cdot \mathrm{s}^{-1}$, the angle of the discharge opening $\beta=60^{\circ}$, the number of pins $z=22 \mathrm{pcs}$. With optimal values of these factors the minimum deviation of feed from the specified rate is $2 \%$.
\end{abstract}

Keywords: compound feed, dosing, individual-dosed distribution, productivity, drum dispenser.

\section{Introduction}

Keeping cattle is associated with high labor costs due to the significant share of manual labor in the process feeding and caring for animals. So, when feeding, only service accounts for up to $60-70 \%$ of the total labor costs [1-3]. One of the labor-intensive operations - the distribution of compound feed to cows, on average, requires about $38 \%$ of the milkmaid's working time. Reduction of these costs is possible only at mechanization and automation of this process for what development of the corresponding technical means is necessary. In [4;5] the authors point out the significant economic effects of introduction of an automatic system for distribution of concentrated feed. Compared to the conventional cattle feeding system, labor efficiency increased by $24 \%$. The total working time of feeding cows in farms with an automatic feeding system decreased by $50.9 \%$ compared to the usual. At the same time, investment in the introduction of automatic feeding can triple. The author in $[6 ; 7]$ indicates a reduction in feed losses during feeding by an average of $50-75 \%$. The main reason for introduction of automatic feeding is reduction of the working hours and workload. During the transition to automatic feeding, the working time was reduced from $2.5 \mathrm{~h} \cdot \mathrm{day}^{-1}$ to $1.02 \mathrm{~h} \cdot \mathrm{day}^{-1}[8 ; 9 ; 10]$. At the same time, the author points to a reduction in the energy consumption per unit of feed from $6.81 \mathrm{~kW} \cdot \mathrm{m}^{-3}$ to $0.76 \mathrm{~kW} \cdot \mathrm{m}^{-3}$ with the introduction of an automatic feeding system. But increased automation and mechanization can lead to increased energy consumption and costs, from $8.8 \mathrm{kWh}$ before feeding automation and $52.6 \mathrm{kWh}$ after automation.

The main structural component of automatic feeding is the design of the dispenser that directly dispenses food. Dispensers must meet the zootechnical conditions for productivity, accuracy and uniformity of dosing, deviation from the specified rate delivery should not exceed $\pm 5 \%$. Existing design solutions for dispensers for dosing feed with differentiated delivery to animals do not fully meet these requirements.

\section{Materials and methods}

Theoretical, experimental and statistical research methods were used in the work. During the experiment and processing of the obtained dependences, experimental planning methods were used. Validation of the obtained dependences of experimental data was carried out by methods of mathematical statistics, using the computer programs "Statistica 6.0", Microsoft Excel.

The developed dispenser (Fig. 1) has a vertical axis of rotation of the drum, the cells of which are made limited on four sides. 


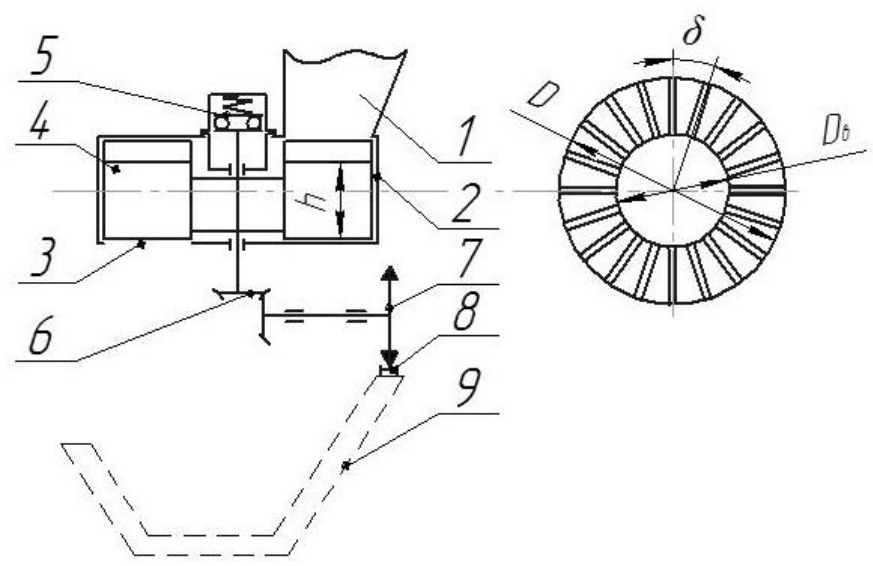

Fig. 1. Structural and functional diagram of the dosing device of the dispenser: 1 - bunker;

2 - case of the batcher; 3 - unloading window; 4 - eye drum; 5 - locking mechanism;

6 - bevel gear; 7 - gear wheel; 8 - drive pins; 9 -feeder

The program of the experimental research provides: determination of physical and mechanical properties of the dosed forage, establishment of dependence productivity, deviation from the norm and non-uniformity of dosing from the basic parameters (number of pins $z$, speed of the feeder $V_{k}$, angle of an unloading opening $\beta$ ).

On the basis of regression analysis mathematical models of the technological process of individual distribution of feed according to certain optimization parameters are developed.

To implement the program of the experimental research, a structural and functional scheme of the dosing device of the feeder was developed (Fig. 1), according to which an installation (Fig. 2) for individual-dosed delivery of compound feed to cows in stalls was made.

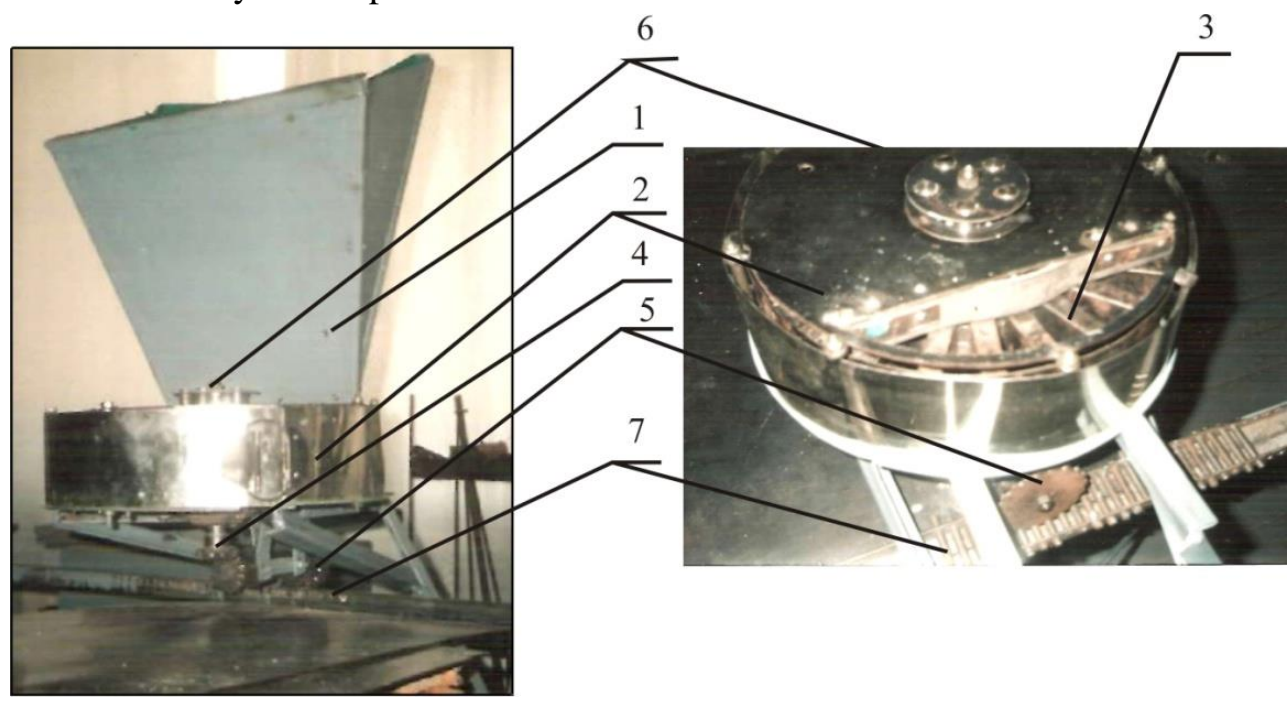

Fig. 2. General view of the experimental setup: 1 - bunker; 2 - dispenser body; 3 - whip drum;

4 - final gear transmission $(i=1) ; 5$ - toothed wheel; 6 - fixed frame mechanism; 7 - pins

The experimental setup includes: an eye drum with a vertical axis of rotation, the eyes which are made limited on four sides, a hopper, a dispenser housing with an unloading window, a locking mechanism, a bevel gear and a gear that contacts the horizontally mounted pins. The dispenser is mounted on a frame that moves on rails. The experimental setup allows to change the number of pins $z$ installed along one feeding front, from 1 to 26 pieces and the speed of movement $V_{k}$ of the experimental setup, to adjust the angle $\beta$ of the unloading hole.

The response criteria are:

- size of the dose $q$ of feed given to one animal, $\mathrm{kg}$;

- deviation from the set rate of feed, $\%$. 
A rotatable noncomposite plan was chosen for the research in order to exclude gross errors (deviations) and to estimate the variance of reproduction three parallel experiments were chosen.

\section{Results and discussion}

This technical solution simplifies the design of the dispenser and reduces energy consumption, as it eliminates the need for a separate electric drive of the dosing device and control mechanism. Compared to existing distributors, it has a number of advantages: in terms of energy and metal consumption, compactness of connection with the feeder allows to increase the dosing accuracy. Improving the dosing accuracy is ensured by matching the gear ratio of the gears and bevel gears with the angle of rotation of the eye drum, as well as the absence of latches that cause delay in the beginning and end of the feed.

During operation of the dispenser, the feed moves along three characteristic zones (Fig. 3b): loading the feed sector between the drum blades, zone I, moving the feed mass with the blade on the stationary bottom of the dispenser zone II, and unloading a portion of feed through the window in the bottom III. After leaving the unloading window, the feed particles enter the feeder.

a)

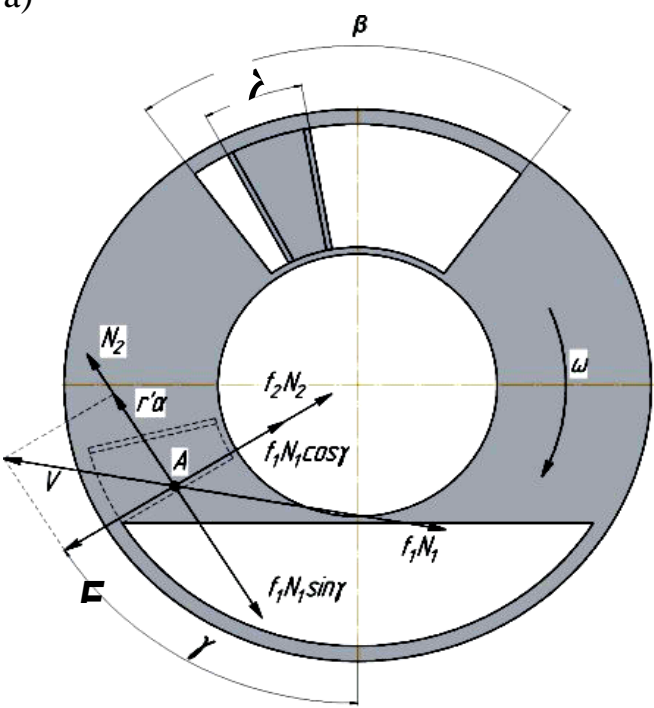

b)

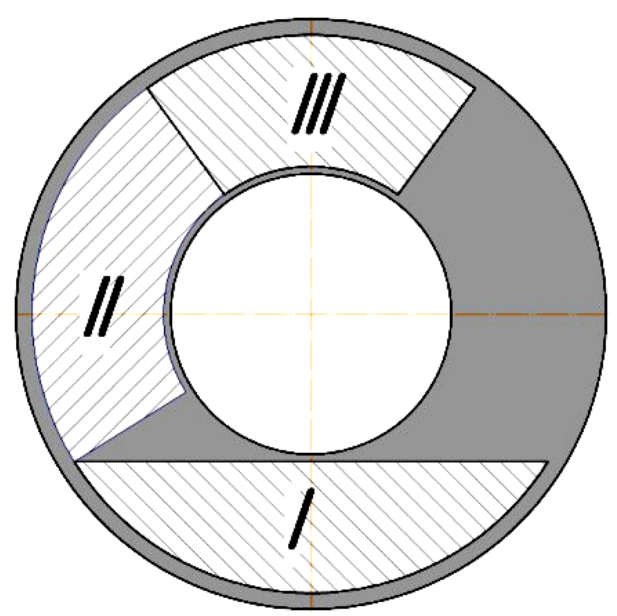

Fig. 3. Schematic diagram of the drum dispenser scheme of acting forces (a) and location of zones (b): $\delta$ - angle of the cell; $\beta$ - angle of the unloading hole; $\gamma$ - angle of rotation of the blade relativeto a fixed position in the direction of the axis; I- loading, II - moving; III - unloading

The movement of feed in the second phase can be considered as a free fall with an initial velocity $V_{0}$ to the bottom of the feeder (Fig. 4). Since the motion in the second phase occurs in the vertical plane, it is advisable to use the Cartesian coordinate system $(\mathrm{x}, \mathrm{y}, \mathrm{z})$.

According to D'alembert's principle, we write down the differential equations of motion of a falling particle

$$
\left.\begin{array}{l}
m \ddot{x}=k V \cos \psi \\
m \ddot{y}=m g-k V \sin \psi
\end{array}\right\},
$$

where $\psi$-angle between the velocity vector at the point and the horizontal coordinate $x$;

$V$ - vertical coordinate;

$k$ - coefficient of proportionality;

$$
k=c \frac{\gamma_{n} F}{g},
$$

where $F$ - midline cross section;

$c$ - constant value with constant midline cross section and particle shape;

$\gamma_{n}-$ air density. 
After a series of substitutions and transformations we obtain equations that allow to determine the values of the parameters required for installation of the dispenser over the feeder: coordinates of feed in the feeder $(2,3)$, particle velocity, fall period (4), and the relative position of the dispenser and feeder in space are defined as the vertical distance between the unloading window of the dispenser and the bottom of the feeder, and horizontally as a projection trajectory of the feed particles when falling on the horizontal axis $x$.

$$
y=\frac{x}{V_{0} \cos \gamma}\left(V_{0} \sin \gamma-\frac{g}{k_{0}}\right)+\frac{g}{k_{0}^{2}} \ln \frac{V_{0} \cos \gamma}{V_{0} \cos \gamma-k_{0} x} .
$$

The obtained trajectory equation allows to determine the distance from the dispenser to the feeder.

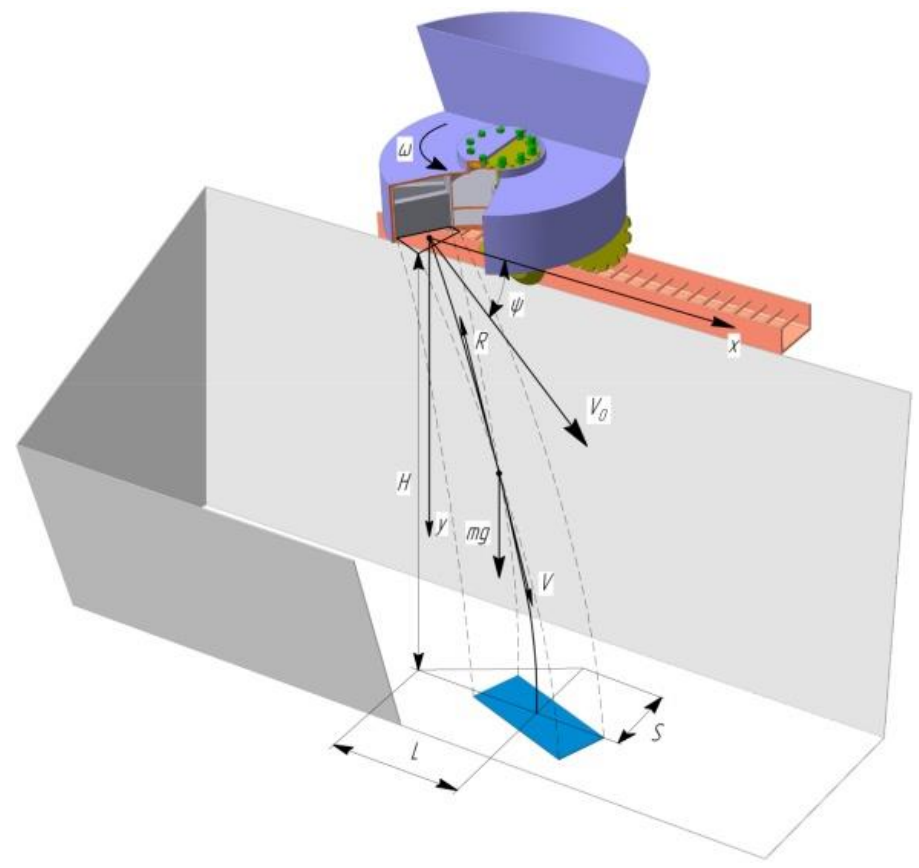

Fig. 4. Scheme of action forces at free falling of forage from the batcher to the bottom of the feeding trough

The change in the $x$ coordinate over time is an exponential dependence. Therefore, the maximum distance from the beginning of the east of the particle and the central zone of the feeding front will be

$$
x_{\max }=\frac{V_{0} \cos \gamma}{k_{0}} \lim _{t \rightarrow \infty}\left(1-e^{-k_{0} t}\right)=\frac{V_{0} \cos \gamma}{k_{0}} .
$$

If the distance $x$ approaches the value $x_{\max }$, it is necessary to significantly raise the dispenser vertically. Taking into account the scattering of the feed width, it is possible to reduce the distance. Then $S=x_{\max }-\Delta x$. and the period of fall $T$ of the feed particle from the feeder to the bottom of the feeder is determined from the equation

$$
T=\frac{1}{k_{0}} \ln \frac{V_{0} \cos \gamma}{V_{0} \cos \gamma-k_{0} S} .
$$

After conducting the experiment and processing the obtained data, using the application program STATISTICA 10.1, regression equations were obtained. Mathematical models after determining the significant regression and decoding coefficients took the form:

- weight of feed given to one cow, $\mathrm{kg}$

$$
q=-0.2951+0.1276 z-0.1375 V+0.0132 \beta,
$$

- deviation from the specified norm, \%

$$
\xi=24.7473-1.7011 z-8.4979 V-0.1479 \beta+0.0387 z^{2}+12.744 V^{2}+0.0011 \beta^{2} .
$$


Analysis of regression equations showed that when the rate of feed is reduced, the difference between the weight feed in one cell and the rate decreases. This, in turn, leads to an increase in the issuance error. When the speed of the distributor increases, the feed from the cells does not have time to wake up during the passage over the unloading hole (a direct relationship between the speed of distributor and the speed of rotation of the drum).

Having determined the partial derivative equations and equating them to zero, we determine the values of the factors that provide the optimal parameters of the process of individually dosed feed. Fig. 5 shows the dependences of the deviation of the feed distribution on the number of pins, the angle of inclination and, accordingly, the speed of movement of the dispenser.
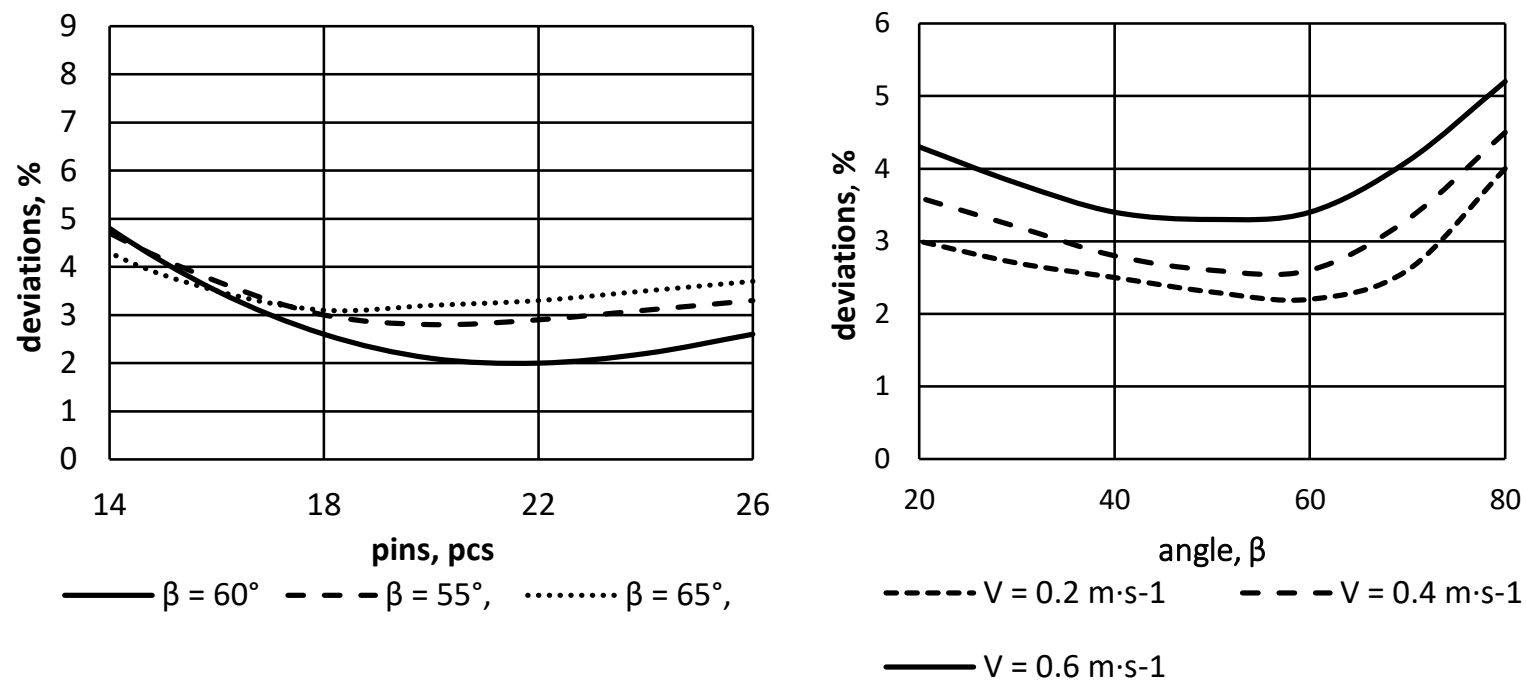

Fig. 5. Influence of the number of pins $z$, speed of the feeder $V$, angle of the unloading hole $\beta$ to deviation of feed from the specified

After analyzing Fig. 5, it becomes clear that the deviation of the feed from the given depends on the combination of rational parameters. The minimum deviation is observed at the number of pins from 18 to 24. In combination with the angle of the unloading hole, the minimum deviation is at an angle of $60^{\circ}$. The dependence of the speed of movement on the angle of the unloading hole indicates the minimum deviation of the feed from the set observed at an angle of 60 for all speeds. At a speed of $0.2 \mathrm{~m}$, this deviation has a minimum value of $2.2 \%$.

For the extremum point, the value of the speed will be equal to $V=0.2 \mathrm{~m} \cdot \mathrm{s}^{-1}$, the angle of the unloading hole $\beta=60^{\circ}$, the number of pins $z=21.98 \mathrm{pcs}$ (as the number of pins can only be an integer value, we take $z=22 \mathrm{pcs}$ ). At the optimal values of these factors, the smallest deviation of the feed from the specified norm will be $2 \%$.

Based on the obtained data, graphs of the influence of two controlled factors on the deviation from the set norm at the optimal value of the third factor were constructed. Analysis of the graphs shows that the total error does not exceed 5\%. That is, the convergence of experimental and theoretical data meets the requirements. Statistical analysis allowed to determine significant regression coefficients and to obtain simplified equations that adequately describe the process with a given $95 \%$ probability.

\section{Conclusions}

1. As a result of the conducted theoretical analysis a rational design and functional scheme of the drum batcher with a vertical axis of rotation for individually-dosed delivery of compound feed, which provides the norm of giving of forage within $1.5 \ldots 3.5 \mathrm{~kg}$ on one cattle place, and deviation from the set norm of delivery to $5 \%$, has been substantiated .

2. Mathematical models of the dispenser are received to carry out analytical research, to check the reliability of the received theoretical dependences, which reflect dependence of qualitative indicators of the process of dosing of compound feeds on the basic constructive parameters of the batcher. 
3. Rational design parameters and mode of operation of the compound feed batcher are determined, experimental researches are carried out.

4. As a result of solving the problem of extremum in relation to the optimization criteria, the rational parameters of the dispenser are determined: the speed value will be equal to $V=0.2 \mathrm{~m} \cdot \mathrm{s}^{-1}$, the angle of the unloading hole $\beta=60^{\circ}$, the number of pins $z=21.98 \mathrm{pcs}$ (as the number of pins can be only an integer, we accept $z=22 \mathrm{pcs}$ ) for these (optimal) values of these factors.

\section{References}

[1] Jinookh O., Riccardoofer H., Tecumseh F. An open source automatic feeder for animal experiments. HardwareX, vol. 1, 2017, pp. 13-21.

[2] Borso F. Influence of automatic feeding systems on design and management of dairy farms. Journal of agricultural engineering, vol. 48, 2017, pp. 48-52.

[3] Mattachini G., Pompe J., Finzi A., Tullo E., Riva. Effects of Feeding Frequency on the Lying Behavior of Dairy Cows in a Loose Housing with Automatic Feeding and Milking System. Animals (Basel), 2019. [online] [21.03.2021] Available at:

https://www.ncbi.nlm.nih.gov/pmc/articles/PMC6523292/

[4] Bisaglia C. Automatic vs. conventional feeding systems in robotic milking dairy farms: a survey in The Netherlands. [online] [21.03.2021] Available at: https://www.researchgate.net/publication/267555832_Automatic_vs_conventional_feeding_s

[5] Sedigh S. Design and build of an automated animal feed dispenser. Available at: https://digitalcommons.calpoly.edu/cgi/viewcontent.cgi?article $=1155 \&$ context $=$ braesp

[6] Navaneeth B., Murtyb A. Automatic poultry feeder. International Journal of Advance Engineering and Research Development, vol. 2, issue 7, 2015, pp. 338-343.

[7] Bisaglia1 C., Belle Z., Berg G. Automatic vs. conventional feeding systems in robotic milking dairy farms: a survey in The Netherlands. [online] [21.03.2021] Available at: https://www.researchgate.net/publication /267555832.

[8] Nabokov V., Novopashin L., Denyozhko L., Sadov A. Applications of feed pusher robots on cattle farmings and its economic efficiency. International Transaction Journal of Engineering, Management, \& Applied Sciences \& Technologies, vol. 11, №.14, 2020, pp. 1-7.

[9] Oberschätzl R., Haidn B., Neiber J., Neser S. Automatic feeding systems for cattle - A study of the energy consumption of the techniques Environmentally friendly agriculture and forestry for future generations. XXXVI CIOSTA CIGR V Conference 2015. Saint Petersburg, the Russian Federation. [online] [21.03.2021] Available at:

https://www.researchgate.net/publication/285592337_Automatic_feeding _systems _for_cattle__A_study_of_the_energy_consumption_of_the_tech.

[10] Pezzuolo A., Chiumenti A., Sartori L. Automatic feeding system: evaluation of energy consumption and labour requirement in north-east italy dairy farm. engineering for rural development. Jelgava, 2016, pp. 882-887. 